\title{
aBoard: uma Plataforma Computacional na Nuvem para Comunicação Alternativa e Educação Inclusiva
}

\author{
Thiago P. Lima, Edson A. Silva, Augusto L. Lima, \\ Natália M. Franco, Robson N. Fidalgo
}

Centro de Informática - Universidade Federal de Pernambuco (UFPE) - Brasil

$$
\{\text { tpl, eas4, all3, nmf,rdnf\}@cin.ufpe.br }
$$

\begin{abstract}
Resumo. A Comunicação Aumentativa elou Alternativa (CAA) é uma Tecnologia Assistiva importante nas adaptações necessárias para pessoas com deficiência na fala (e.g., Paralisia Cerebral e Autismo). Neste contexto, apresentase a Plataforma aBoard para CAA e educação inclusiva, a qual tem os seguintes diferenciais: arquitetura em nuvem, sugestão de pictogramas e respostas rápidas. O uso desta plataforma traz um conjunto de benefícios à educação e à saúde dessas pessoas. De forma geral, o uso do aBoard favorece a interação com outras pessoas, o desenvolvimento de habilidades cognitivas, a redução de frustrações e a melhoria da autoestima e da qualidade de vida.
\end{abstract}

\section{Cenário de uso}

Segundo dados do Censo 2010 do IBGE [IBGE 2010], exitem 2,6 milhões de pessoas com alguma deficiência intelectual (e.g., Transtorno do Espectro Autista e Síndrome de Down) ou desordens neurológicas (e.g., Paralisia Cerebral, Lesão Crânio Encefálica e Microcefalia) que podem ter sua capacidade de comunicação limitada de forma temporária ou permanente. Vale ressaltar que estes dados estão defasados e não contemplam, por exemplo, os casos do surto de microcefalia ocorridos no Brasil entre 2015 e 2016.

De maneira geral, a privação da comunicação restringe as possibilidades de desenvolvimento do indivíduo, pois este não consegue ter acesso igualitário à educação e às oportunidades de crescimento intelectual. Em um contexto mais específico, quando a privação da comunicação ocorre na infância, esta pode provocar déficit no desenvolvimento cognitivo e atraso na aquisição da língua escrita [Mousinho et al. 2008]. Para estes casos, faz-se necessária a inserção de recursos de Tecnologia Assistiva (TA) que visam complementar e/ou substituir a comunicação destes indivíduos, os quais são denominados recursos de Comunicação Aumentativa e/ou Alternativa (CAA).

No que tange à educação, as classes comuns do ensino regular não proveem assistência compatível com a necessidade desses indivíduos. Assim, a fim de melhorar a participação social, o desenvolvimento intelectual e o crescimento educacional dessas pessoas, em 2009, o Ministério da Educação instituiu que os alunos com deficiência devem ser matriculados nas classes comuns do ensino regular, bem como no Atendimento Educacional Especializado (AEE) [BRASIL 2009]. O AEE tem como função, complementar ou suplementar a formação do aluno por meio da disponibilização de recursos que eliminem as barreiras para sua plena participação na sociedade e desenvolvimento de sua aprendizagem.

A fim de desenvolver o potencial destas pessoas, o governo federal tem investido bastante na educação inclusiva e em recursos para o AEE, a saber: 1) Entre 2014 e 2015, 
a prefeitura do Recife investiu cerca de $\mathrm{R} \$ 8$ milhões para adquirir tablets e 5 mil licenças de um software para Comunicação Alternativa ${ }^{1,2}$; e 2) Em 2016, o governo do estado do Paraná investiu cerca de $\mathrm{R} \$ 76,2$ milhões na educação especial ${ }^{3}$. Estes números mostram a importância desta área, porém apesar dos investimentos, ainda há muito o que se fazer, pois a compra de licenças de software não é uma realidade para municípios de menor arrecadação, tampouco para as famílias das pessoas com deficiência.

Com o propósito de prover uma ferramenta para o AEE que se adeque às necessidades das pessoas com deficiência na fala, apresenta-se o aBoard, uma plataforma computacional gratuita e em nuvem para CAA e educação inclusiva. Na Figura 1-A apresenta-se uma visão geral da Plataforma aBoard, a qual é baseada em três componentes: aBoard, aBoard Editor e aBoard Server. O aBoard é um aplicativo de CAA para dispositivo móvel que tem como principais usuários as pessoas com deficiência na fala (i.e., pessoas com TEA, PC ou Microcefalia). Destaca-se que este aplicativo pode ser utilizado imediatamente após a instalação, uma vez que vem com um vocabulário de 260 palavras prontas para uso geral. Já o aBoard Editor, é um sistema Web desenvolvido para personalizar o conteúdo (vocabulário e atividades pedagógicas) utilizado no aplicativo e tem como principais usuários os mediadores (e.g., familiares e profissionais de educação/saúde) das pessoas com deficiência na fala. O aBoard Editor disponibiliza em torno de 15 mil pictogramas (i.e. símbolos que representam objetos ou conceitos por meio de desenhos figurativos) do ARASAAC [Palao 2017] para a personalização do conteúdo. Por fim, o aBoard Server é um serviço em nuvem responsável pelo armazenamento dos dados, disponibilização de conteúdo e controle de acesso dos usuários.

Dado que a personalização do vocabulário é guiada pela criatividade do mediador, a Plataforma aBoard pode ser usada pelos professores de sala de aula e do AEE para diversos fins educacionais, tais como:

- Adaptação de conteúdo pedagógico - pode-se adaptar o vocabulário para diversas atividades educacionais, por exemplo, para o ensino de vogais, consoantes, sílabas, números e operações aritméticas, bem como para o ensino de outros conceitos como, por exemplo, animais, cores, lugares, instrumentos musicais). Na Figura 1-B mostra-se um exemplo de adaptação de conteúdo para o ensino das letras do alfabeto;

- Atividades lúdicas - pode-se usar os pictogramas para contar e produzir histórias colaborativas com os alunos, bem como trabalhar conceitos presentes em músicas infantis;

- Instrução de rotinas - pode-se usar os pictogramas para ensinar rotinas, tais como: "escovar os dentes", "tomar banho" e "ir para a escola".

Pelo fato da personalização de conteúdo poder ser feita fora do dispositivo móvel e na nuvem, a Plataforma aBoard permite que esta atividade seja realizada a partir de qualquer computador com acesso à Internet e sem tirar o dispositivo móvel do usuário. Outrossim, mesmo que o computador ou o dispositivo móvel sejam danificados, o conteúdo

\footnotetext{
${ }^{1}$ http://jconline.ne10.uol.com.br/canal/cidades/educacao/noticia/2015/06/09/aplicativo-vai-ajudaralunos-com-necessidades-especiais-das-escolas-municipais-do-recife-184864.php

${ }^{2}$ http://www1.folha.uol.com.br/empreendedorsocial/2016/11/1826931-aplicativo-livox-da-voz-eautonomia-para-pessoas-com-deficiencias.shtm

${ }^{3} \mathrm{http}: / /$ www.aen.pr.gov.br/modules/noticias/article.php?storyid=92833\&tit=Parana-e-o-segundoEstado-que-mais-investiu-em-educacao-em-2016
} 

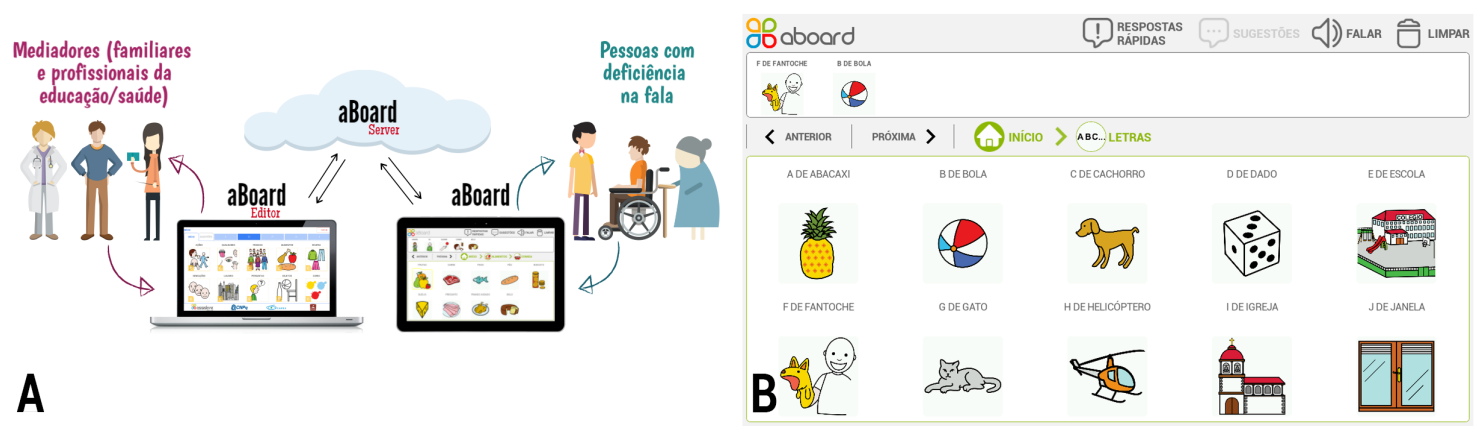

A

(B) Atividade pedagógica adaptada

guardado na nuvem sempre poderá ser acessado a partir de qualquer computador ou dispositivo móvel. Vale destacar que a personalização de conteúdo requer acesso constante à Internet, enquanto que a utilização desses conteúdos no dispositivo móvel pode ser feita em modo offline, pois a Internet só é necessária no momento de sincronização do conteúdo. Ressalta-se ainda que o processo de personalização e sincronização de conteúdo é simples e rápido. Por exemplo, durante uma atividade de contação de histórias, pode-se inserir um novo personagem com pouco tempo e esforço no aBoard Editor e, depois, sincronizá-lo com o aBoard em apenas 2 cliques no aplicativo.

Em suma, a Plataforma aBoard é adaptável para diversos fins educacionais e pode ser utilizada com crianças que tenham alguma limitação na fala. O limite desta tecnologia é a criatividade e o empenho do professor em adaptar conteúdos para explorar o potencial e contribuir para o desenvolvimento destas crianças. A ideia é que esta tecnologia sirva como um apoio para o desenvolvimento inicial da criança e que, com o passar do tempo, esta criança possa evoluir de forma a se tornar independente deste apoio tecnológico. Porém, vale ressaltar que existem casos mais severos em que a criança não consegue atingir esta independência e permanece utilizando a tecnologia durante toda a vida.

\section{Desenvolvimento}

O processo de desenvolvimento escolhido foi a abordagem iterativa e incremental, passando por ciclos de análise, projeto, implementação e testes [Sommerville et al. 2003]. Esta abordagem foi escolhida por permitir a entrega rápida de versões e facilitar a avaliação dos aplicativos com o usuário final. Na Figura 2 apresenta-se os principais componentes da plataforma aBoard.

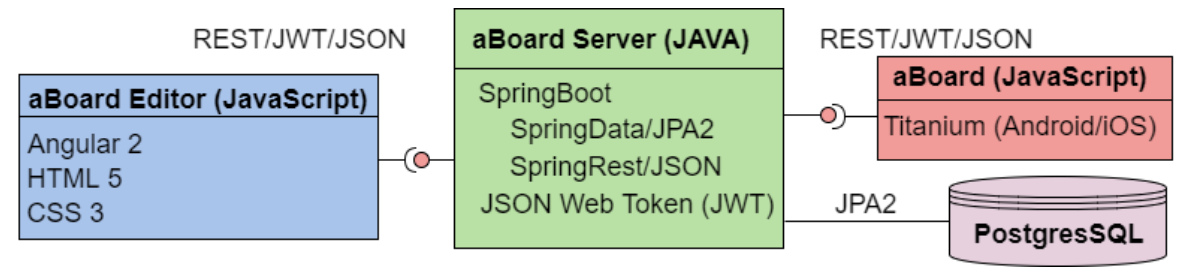

Figura 2. Principais componentes da Plataforma aBoard

O componente aBoard é desenvolvido utilizando a linguagem JavaScript e o framework Titanium. O componente aBoard Editor é desenvolvido utilizando o framework Angular 2 e segue as especificações HTML5 e CSS3. E, o componente aBoard Server é 
VI Congresso Brasileiro de Informática na Educação (CBIE 2017)

Anais dos Workshops do VI Congresso Brasileiro de Informática na Educação (WCBIE 2017)

desenvolvido utilizando a linguagem JAVA e tem como base os frameworks SpringBoot - encarregado por gerenciar o ciclo de vida da aplicação -, SpringData - responsável por gerenciar o acesso ao banco de dados (i.e., PostgresSQL) por meio da especificação Java Persistence API 2 (JPA2) - e SpringRest - ajuda a criar serviços Web usando os padrões Representational State Transfer (REST) e JavaScript Object Notation (JSON). Para o controle de acesso, é implementado o padrão JSON Web Token (JWT). A avaliação da plataforma, realizada a cada ciclo de desenvolvimento, consiste em duas etapas: a primeira, no decorrer da implementação, usando testes automatizados e, a segunda, com auxílio do usuário final.

\section{Apresentação da Plataforma aBoard}

\subsection{Aplicativo aBoard}

Na Figura 3-A mostra-se a interface principal do aBoard. Nesta figura pode-se ver os seguintes componentes gráficos: 1) Botão aBoard; 2) Botão Respostas Rápidas; 3) Botão Sugestões; 4) Botão Falar; 5) Botão Limpar; 6) Barra de expressão; 7) Botão para rolagem à direita; 8) Botão para rolagem à esquerda; 9) Barra de localização e navegação e 10) Área de conteúdo.
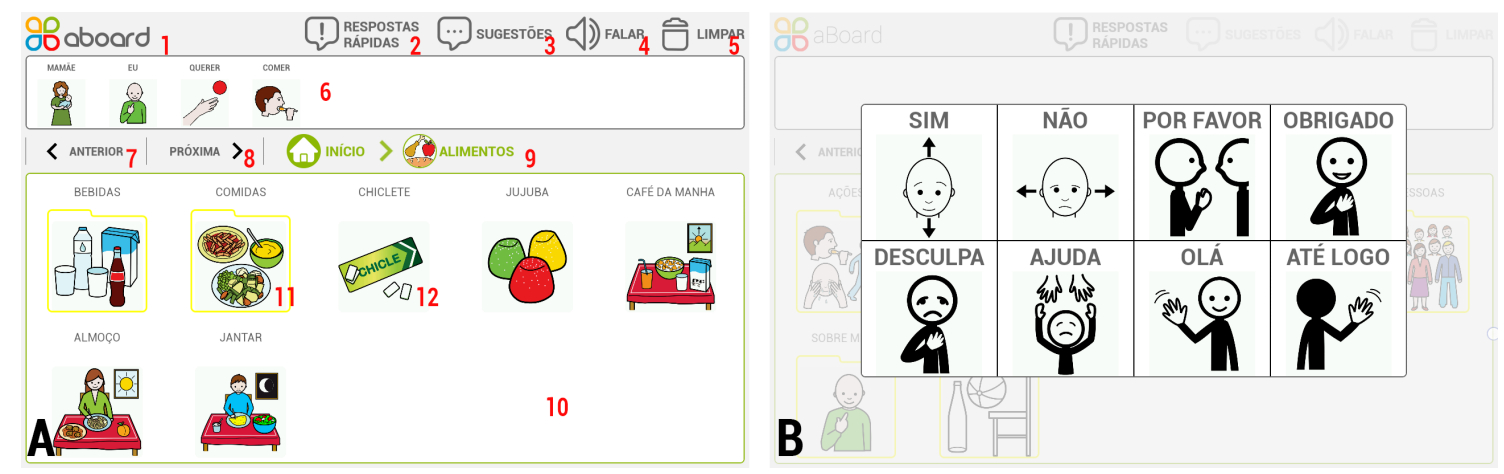

Figura 3. (A) Interface do aBoard e (B) Respostas rápidas do aBoard

Os pictogramas que formam o vocabulário são organizados a partir dos conceitos de categorias e elementos, os quais remetem à ideia de pastas e arquivos de um computador. Ou seja, assim como os arquivos são organizados em pastas, os elementos são organizados em categorias. Por exemplo, o elemento BOLO pode ser inserido na categoria COMIDAS, bem como o elemento LEITE pode ser inserido na categoria BEBIDAS. Neste contexto, assim como pastas podem ser criadas dentro de outras pastas, COMIDAS e BEBIDAS podem ser criadas dentro de uma categoria ALIMENTOS. Ou seja, COMIDAS e BEBIDAS são subcategorias de ALIMENTOS. Vale ressaltar que existem duas categorias especiais: AÇÕES e QUALIDADES, as quais são usadas para organizar verbos e adjetivos, respectivamente. Para diferenciar uma categoria de um elemento, usa-se uma moldura amarela em formato de pasta para representar uma categoria e nenhuma moldura para indicar que se trata de um elemento.

Uma expressão pode ser criada a partir da seleção de um conjunto de no máximo 10 (dez) pictogramas. Para selecionar um elemento, deve-se navegar até a sua categoria. Por exemplo ${ }^{4}$, para selecionar o elemento BOLO deve-se dar um toque simples na

\footnotetext{
${ }^{4}$ Isso depende de como o vocabulário foi personalizado
} 
VI Congresso Brasileiro de Informática na Educação (CBIE 2017)

Anais dos Workshops do VI Congresso Brasileiro de Informática na Educação (WCBIE 2017)

categoria ALIMENTOS, outro na categoria COMIDAS e, por fim, um toque simples no elemento BOLO. Caso o elemento desejado não apareça entre os 10 primeiros elementos da categoria, usa-se os botões ANTERIOR e PRÓXIMA para rolar, respectivamente, à esquerda ou à direita, ou desliza-se a mão no sentido horizontal, até que o elemento desejado seja encontrado. Vale ressaltar que as categorias não aparecem na barra de expressão e estas existem apenas para organizar o conteúdo a ser exibido. Para agilizar a produção da expressão, pode-se dar um toque simples no botão SUGESTÕES para solicitar a sugestão de um elemento que complete o sentido do último elemento selecionado. Por exemplo, é possível ter as seguintes sugestões para BOLO: COMER, QUERER, GOSTOSO e RUIM. Recomenda-se o uso da barra de navegação (breadcrumb) quando se deseja navegar para uma categoria anterior sem ter que ir para a tela inicial. Por exemplo, considerando a categorização INÍCIO $\rightarrow$ ALIMENTOS $\rightarrow$ COMIDAS, é possível ir diretamente para a categoria ALIMENTOS, sem ter que ir para a tela inicial (i.e., INÍCIO). Como estímulo auditivo, todo elemento ou categoria selecionado tem a sua legenda falada automaticamente.

Para falar a expressão criada, basta dar um toque simples no botão FALAR. Além desta opção, como reforço auditivo, cada elemento da expressão também pode ser falado individualmente. Para isto, basta dar um toque simples sobre o elemento que compõe a expressão. Se durante a produção da expressão houver a necessidade de dar uma resposta rápida sem interferir na produção da mesma, basta dar um toque simples no botão RESPOSTAS RÁPIDAS e escolher uma das opções exibidas na Figura 3-B. Para sair desta tela sem escolher uma opção, deve-se tocar em qualquer parte opaca da tela. Para limpar uma expressão toda, deve-se dar um toque simples no botão LIMPAR. Por outro lado, caso haja interesse em excluir apenas um elemento específico da expressão, deve-se dar um toque longo sobre o elemento que deseja excluir. Toda vez que o conteúdo for personalizado no aBoard Editor, deve-se atualizá-lo no aBoard. Para isto, deve-se dar um toque no menu aBoard e depois no botão ATUALIZAR ABOARD. Diferentemente das outras ações, esta requer uma conexão de Internet para funcionar. Para sair do aplicativo, deve-se tocar no menu aBoard e depois no botão SAIR DA CONTA.

\section{2. aBoard Editor}

Na Figura 4-A mostra-se a tela do aBoard Editor, responsável pela personalização do conteúdo do aBoard. Nesta figura é possível ver os seguintes componentes gráficos: 1) Barra de navegação; 2) Botão para sair do sistema; 3) Aba de conteúdo; 4) Aba de sugestões; 5) Botão rolar à esquerda; 6) Botão criar; 7) Botão editar; 8) Botão excluir; 9) Botão rolar à direita e 10) Área de conteúdo.

É possível utilizar o aBoard Editor para personalizar o conteúdo como desejado, porém, a fim de desenvolver pensamentos abstratos e ensinar generalizações de conceitos, recomenda-se nomear as categorias usando legendas que representem conceitos (e.g., ALIMENTOS, COMIDAS e BEBIDAS) ao invés de contextos de uso (e.g., COZINHA, PARQUE e ALMOÇO). Não há restrições para criar categorias ou subcategorias. Contudo, a fim de reduzir a quantidade de cliques para encontrar os elementos, recomenda-se evitar hierarquias de categorias com mais de 3 (três) níveis. Por exemplo, evite hierarquias como: ALIMENTO $\rightarrow$ COMIDA $\rightarrow$ CARNE $\rightarrow$ BRANCA $\rightarrow$ AVE.

Para diferenciar categorias e elementos, as categorias são representadas visualmente pela letra "C". Por sua vez, os elementos são representados pelas letras "A", "Q" 


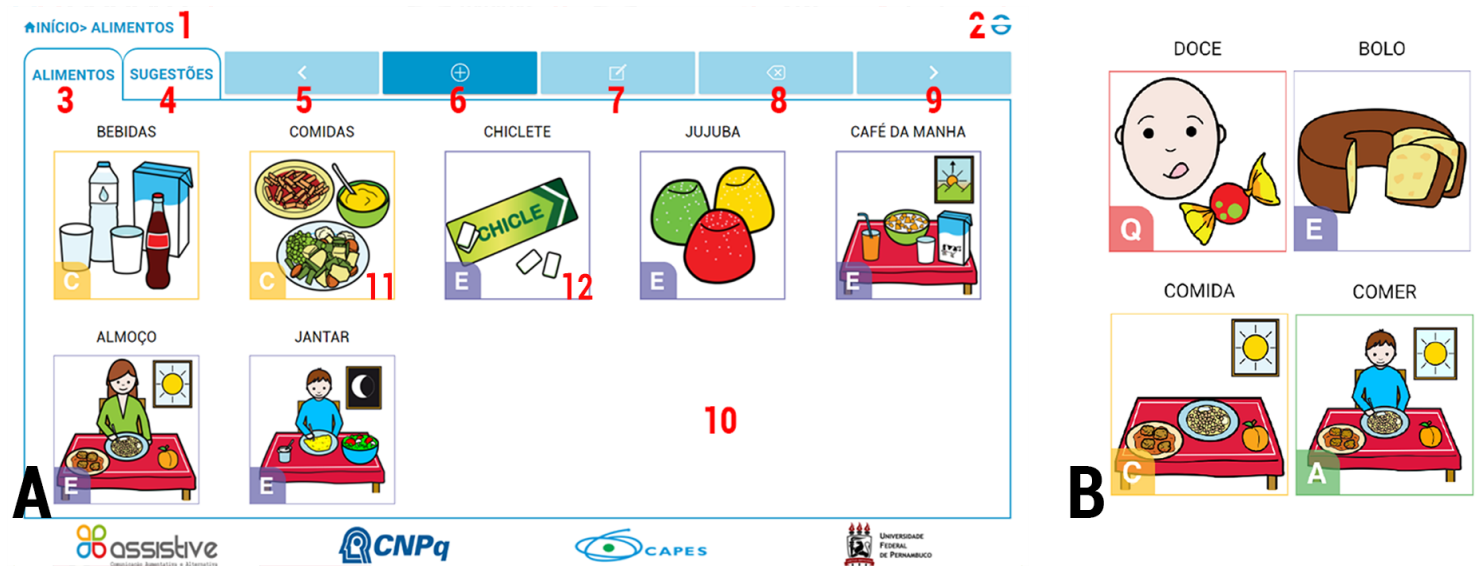

Figura 4. (A) Interface do aBoard Editor e (B) Tipos de pictogramas no aBoard Editor

e "E", as quais informam se o elemento é uma ação, uma qualidade ou um elemento comum. Além destas representações, são usadas bordas com cores diferentes para distinguir categorias de elementos. As categorias têm borda amarela e os elementos têm borda verde (ação), vermelha (qualidade) ou azul (comum). Na Figura 4-B mostram-se estas representações.

A criação de uma categoria ou elemento segue os seguintes passos: 1) Decidir onde a categoria ou elemento será criado. Por exemplo, para criar a categoria COMIDAS dentro da categoria ALIMENTOS, deve-se estar dentro da categoria ALIMENTOS. Para criar o elemento BOLO dentro da categoria COMIDAS, deve-se estar dentro da categoria COMIDAS; 2) Clicar no botão para entrar no modo de criação; 3) Informar se deseja criar uma categoria ou elemento; 4) Clicar no botão para buscar um pictograma; 5) Escrever uma palavra chave para buscar um pictograma; 6) Escolher um pictograma dentre os vários retornados; 7) Editar a legenda do pictograma escolhido (este passo é opcional); e 8) Clicar no botão Salvar para confirmar ou no botão Cancelar para anular a criação.

A edição de uma categoria ou elemento segue os seguintes passos: 1) Clicar na categoria ou elemento que deseja editar para selecioná-lo; 2) Clicar no botão para entrar no modo de edição; 3) Clicar na imagem do pictograma caso queira mudá-la; 4) Clicar na legenda caso queira trocá-la; e 5) Clicar no botão Salvar para confirmar ou no botão Cancelar para anular a edição.

A exclusão de uma categoria ou elemento segue os seguintes passos: 1) Clicar na categoria ou elemento que deseja excluir para selecioná-lo; 2) Clicar no botão para entrar no modo de exclusão; e 3) Clicar no botão Excluir para confirmar ou no botão Cancelar para anular a exclusão.

Para agilizar a construção de expressões, é possível sugerir ações ou qualidades como complementos dos elementos de uma categoria. Por exemplo, é possível sugerir as qualidades GOSTOSO e RUIM, bem como as ações QUERER e GOSTAR para a categoria ALIMENTOS, permitindo que todos os seus elementos tenham estas sugestões. Assim, considerando BOLO como um elemento da categoria ALIMENTOS, as ações e qualidades acima são sugeridas como complementos válidos para BOLO. Vale ressaltar que as sugestões de uma categoria também valem para os elementos de suas subcatego- 
VI Congresso Brasileiro de Informática na Educação (CBIE 2017)

Anais dos Workshops do VI Congresso Brasileiro de Informática na Educação (WCBIE 2017)

rias. Assim, se o elemento BOLO estiver na subcategoria COMIDAS, todas as vezes que forem solicitadas sugestões para este elemento, irão aparecer as sugestões da categoria ALIMENTOS mais as sugestões da subcategoria COMIDAS. Destaca-se que elementos semitransparentes correspondem às sugestões que vêm de categorias superiores. Para indicar uma sugestão faça os passos a seguir: 1) Entrar na categoria desejada; 2) Clicar na aba SUGESTÕES; 3) Clicar no botão para indicar que vai fazer uma sugestão; 4) Informar se deseja indicar uma qualidade ou ação; 5) Escrever uma palavra chave para buscar a sugestão desejada; e 6) Selecionar a qualidade ou ação a ser sugerida. Para desfazer uma sugestão faça os seguintes passos: 1) Entrar na categoria desejada; 2) Clicar na aba SUGESTÕES; 3) Clicar no elemento que deseja tirar da lista de sugestões para selecionálo; 4) Clicar no botão para indicar que vai desfazer uma sugestão; e 5) Clicar no botão Desfazer para confirmar a ação ou no botão Cancelar para anular a ação.

\section{Considerações finais}

As classes comuns do ensino regular não têm recursos tecnológicos suficientes para incluir e desenvolver o potencial das pessoas com alguma limitação na fala. A fim de melhorar a participação social e o desenvolvimento destes indivíduos, o Ministério da Educação instituiu o AEE, que tem como função complementar ou suplementar a formação do aluno por meio da disponibilização de recursos que eliminem as barreiras para sua plena participação na sociedade e desenvolvimento intelectual.

Neste contexto, este artigo apresenta a Plataforma aBoard (aBoard, aBoard Editor e aBoard Server) para CAA e educação inclusiva que: 1) É disponível offline, não precisa conexão com a Internet para utilizar o dispositivo; 2) Tem uma interface minimalista, o que mantém o foco do usuário no que é importante - os pictogramas; 3) Facilita a busca por pictogramas e ensina conceitos de abstração e ligações semânticas, dada à categorização do vocabulário e a função de sugestões; 4) É em nuvem, permitindo que o usuário tenha acesso aos seus dados a partir de qualquer dispositivo móvel ou computador; 5) Provê feedback visual e sonoro, que funciona como um reforço para o usuário; 6) Permite a criação explícita de expressões, com a possibilidade de vocalizar cada pictograma da expressão ou a expressão toda; 7) Tem recurso para respostas rápidas (e.g., Sim, Não, Obrigado, Ajuda), o que encoraja o diálogo com outras pessoas; e 8) Permite a personalização do vocabulário, o que aumenta a identificação entre o usuário com deficiência na fala e o dispositivo, encorajando a sua adoção, e permite sua adequação para diversos fins educacionais, tais como adaptação de conteúdos pedagógicos, contação de histórias e instrução de rotinas. Ressalta-se que apesar de existirem outras soluções para CAA, a Plataforma aBoard se diferencia destas porque traz 3 contribuições inovadoras para a área de CAA, a saber: solução em nuvem, sugestões e respostas rápidas.

O uso da Plataforma aBoard traz um conjunto de benefícios à educação e à saúde das pessoas com deficiência na fala, favorecendo: 1) A interação com outras pessoas Com o aBoard a pessoa com deficiência na fala pode dialogar com outras pessoas a partir de um canal alternativo àquele que está impedido; 2) $\mathrm{O}$ desenvolvimento de habilidades cognitivas - Com o aBoard pode-se trabalhar habilidades referentes à percepção, atenção, memória (imediata, recente/mediata, remota, visual, auditiva e viso motora), raciocínio, conceituação e linguagem; 3) A adaptação de conteúdos para a educação inclusiva - Dada a capacidade de personalização de conteúdo, o aBoard pode ser usado para a adaptação de diversos conteúdos pedagógicos, desde atividade lúdicas até a alfabetização; 
4) $\mathrm{O}$ ensino de rotinas - Pessoas com comprometimento cognitivo precisam de um apoio específico para aprender rotinas de saúde, educacionais e familiares; 5) A redução de frustrações - O simples fato de poder comunicar o que deseja, seus pensamentos, sentimentos e necessidades, de forma mais clara e, principalmente, ser entendido, reduz o nível de frustração dessas pessoas; 6) A melhoria da autoestima e da qualidade de vida A comunicação é fundamental para a vida em sociedade e, quando esta é efetiva, empodera a pessoa com deficiência, aumentando sua autoestima e melhorando sua qualidade de vida; e 7) Sua divulgação e adoção - Por ser uma solução gratuita, a plataforma aBoard pode ser amplamente divulgada e adotada por diversos setores públicos, bem como por familiares de pessoas com deficiência.

O aplicativo aBoard foi disponibilizado, de maneira gratuita e ilimitada, no dia 2 de abril de 2017. Este pode ser baixado por usuários Android direto do Google Play (https://play.google.com/store/apps/details?id=assistive.aboard), onde contabiliza-se de 1.000 a 5.000 instalações e nota 4,9. Por sua vez, o aBoard Editor pode ser acessado pelo link http://assistive.cin.ufpe.br/editor.

Como projetos futuros, já estão em desenvolvimento um acionador externo - para pessoas que tenham alguma limitação motora que as impeça de utilizar um dispositivo móvel -, a versão IOS do aBoard e um módulo de predição de pictogramas que considera os aspectos sintáticos e semânticos das frases produzidas. Vale ressaltar que, o objetivo deste módulo é servir de apoio ao letramento e à alfabetização, uma vez que ensina aos alunos a maneira correta de estruturar as frases.

\section{Agradecimentos}

Esta pesquisa é suportada pelo CNPq (458798/2013-4 e 461492/2014-8) e pela Capes (88887.095638/2015-01).

\section{Referências}

BRASIL (2009). Resolução no 4, de 2 de Outubro de 2009. Institui Diretrizes Operacionais para o Atendimento Educacional Especializado na Educação Básica, modalidade Educação Especial.

IBGE, C. D. (2010). Características gerais da população, religião e pessoas com deficiência. Rio de Janeiro: Instituto Brasileiro de Geografia e Estatística.

Mousinho, R., Schmid, E., Pereira, J., Lyra, L., Mendes, L., and Nóbrega, V. (2008). Aquisição e desenvolvimento da linguagem : dificuldades que podem surgir neste percurso. Revista de Psicopedagogia, 25(78):297-306.

Palao, S. (2017). ARASAAC - Portal Aragonês de Comunicação Aumentativa e Alternativa. http://catedu.es/arasaac/.

Sommerville, I., Melnikoff, S. S. S., Arakaki, R., and de Andrade Barbosa, E. (2003). Engenharia de software, volume 6. Addison Wesley São Paulo. 